\title{
Validation of a solid-state anomaloscope used to assess red-green color vision defects
}

\author{
GEORGE A. GERI \\ University of Dayton Research Institute, Williams Air Force Base, Arizona \\ and \\ DAVID F. NERI \\ Naval Submarine Medical Research Laboratory, Groton, Connecticut
}

\begin{abstract}
Solid-state anomaloscopes whose stimuli are derived from light-emitting diodes (LEDs) are simpler and less expensive than conventional anomaloscopes. We have assessed the test-retest reliability and the validity of one solid-state anomaloscope and have obtained normative data for it. Reliability and validity were assessed by classifying 36 color-defective observers into one of five categories defined by degree of defect. When all color defectives were considered, both the validity and reliability of the solid-state anomaloscope were found to be high. The primary stimuli of the solid-state anomaloscope are less well separated in chromaticity space than are those of conventional anomaloscopes, but there is no evidence that this results in the incorrect classification of anomalous trichromats as dichromats. The solid-state anomaloscope appears to be an acceptable alternative to standard anomaloscopes for both research and screening applications.
\end{abstract}

An anomaloscope is a device with which an observer makes a visual match to a given test light by adjusting the proportion of two appropriately chosen primary lights. In most anomaloscopes, the primary lights are red and green and the test light is yellow. This choice was influenced by the observation of Lord Rayleigh (1881) that the proportion of red to green required to match a given yellow can vary substantially from person to person and may be used to identify individuals with abnormal color vision. Despite the unique value of anomaloscopes for assessing color vision defects, other testing devices are easier to obtain and to use and are often substituted for anomaloscope testing. However, many of these devices can distinguish only relatively broad classes of color defect, and the result often is an incomplete, and hence an inaccurate, classification.

Anomaloscopes are also not widely used because of their high cost, which is due in part to the mechanical and optical complexity required to produce bright narrow bandwidth lights and to present them in a controlled manner. This complexity makes maintenance and calibration of the instrument difficult and further discourages its

This research was supported by the Air Force Office of Scientific Research (Life Sciences Task 2313T3), by Air Force Contract F-3361584-C-0066 (UDRI), and by Naval Medical Research and Development Command Work Unit 65850N-M0100-M0100001-1023. We thank R. T. Kintz for the loan of his anomaloscope, P. A. Wetzel for his help in evaluating the timer circuitry, and S. M. Luria for his critical reading of the manuscript.

The authors' mailing addresses are: G. Geri, University of Dayton Research Institute, Higley, AZ 85236-0044, and D. Neri, Naval Submarine Medical Research Laboratory, Naval Submarine Base, Groton, CT 06349-5900. use. Recently, Kintz (1983) described a solid-state anomaloscope in which light-emitting diodes (LEDs) are used as stimuli, thereby reducing the expense and complexity of light source regulation and wavelength control (Piantanida, 1976; White, Wolbarsht, \& Tieger, 1975). Furthermore, because the LEDs are in the form of bicolor light bars, the optical alignment problems of other solid-state anomaloscopes (Dain, Strange, \& Boyd, 1980; Saunders, 1976) are avoided. Thus, the Kintz anomaloscope is a compact, portable, and inexpensive instrument that appears suitable for classifying red-green color defects. Before such an instrument can be adopted for general use, however, it must be established that classifications obtained using it are comparable to those obtained with more conventional instruments.

We report here an evaluation of the reliability and validity of the Kintz anomaloscope. As suggested by the NRC Committee on Vision (1981), reliability was judged by test-retest data and validity by comparison with a standard Nagel anomaloscope. In addition, normative data for the Kintz anomaloscope were obtained from a group of normal trichromats so that we could establish the distribution of matching midpoints (MMPs) and matching ranges (MRs) needed for classifying the color-defective observers.

\section{METHOD}

\section{Observers}

Data were obtained from 20 deutans, 16 protans, and 83 normal trichromats. Most of the color-defective observers were recruited from the Hartford, Connecticut, area through newspaper advertisements and were paid $\$ 7$ 
per hour. Six color-defective observers and all of the normal trichromats were recruited from among the military and civilian personnel at either the Naval Submarine Base (Connecticut) or Williams Air Force Base (Arizona) and were not paid for their participation.

\section{Apparatus}

The solid-state anomaloscope, which is the object of this study, was described by Kintz (1983). ${ }^{1}$ The anomaloscope comparison and primary lights were derived from Hewlett-Packard LED bicolor light bars, the yellow comparison light from one half of an HLMP-2950, and the primaries from an HLMP-2965. The peak wavelengths of the yellow, green, and red stimuli were $583 \mathrm{~nm}$, $565 \mathrm{~nm}$, and $635 \mathrm{~nm}$, respectively, and their halfamplitude bandwidths were $37 \mathrm{~nm}, 28 \mathrm{~nm}$, and $41 \mathrm{~nm}$, respectively. A Nagel Model I anomaloscope was used as the comparison standard. The circular bipartite field for both anomaloscopes was about $1.75^{\circ}$ in diameter.

The chromaticities of the primary stimuli of both the Nagel and Kintz anomaloscopes were measured using a Photo Research (Model PR-703A) Fast Spectral Scanner. The chromaticities associated with selected scale readings of both anomaloscopes are shown in Figure 1, along with a portion of the spectrum locus of the CIE 1976 UCS diagram. The MR data of the present study are expressed as distances, in UCS space, defined as:

$$
\Delta \mathrm{E}=\left[\left(u^{\prime}{ }_{1}-u^{\prime}{ }_{2}\right)^{2}+\left(v^{\prime}{ }_{1}-v^{\prime}{ }_{2}\right)^{2}\right]^{1 / 2},
$$

where $\left(u_{1}^{\prime}, v_{1}^{\prime}\right)$ and $\left(u_{2}^{\prime}, v_{2}^{\prime}\right)$ are the chromaticity coordinates of the MR endpoints. The MMP was then taken as the chromaticity coordinate $\left[\left(u^{\prime}{ }_{1}+u^{\prime}{ }_{2}\right) / 2,\left(v^{\prime}{ }_{1}+v_{2}{ }_{2}\right) / 2\right]$. For the Nagel anomaloscope, the luminances of the primary stimuli varied from 2.7 to $6.9 \mathrm{~cd} / \mathrm{m}^{2}$ from the red to green extremes of the scale, whereas for the Kintz anomaloscope the analogous range was 7.5 to $27.7 \mathrm{~cd} / \mathrm{m}^{2}$.

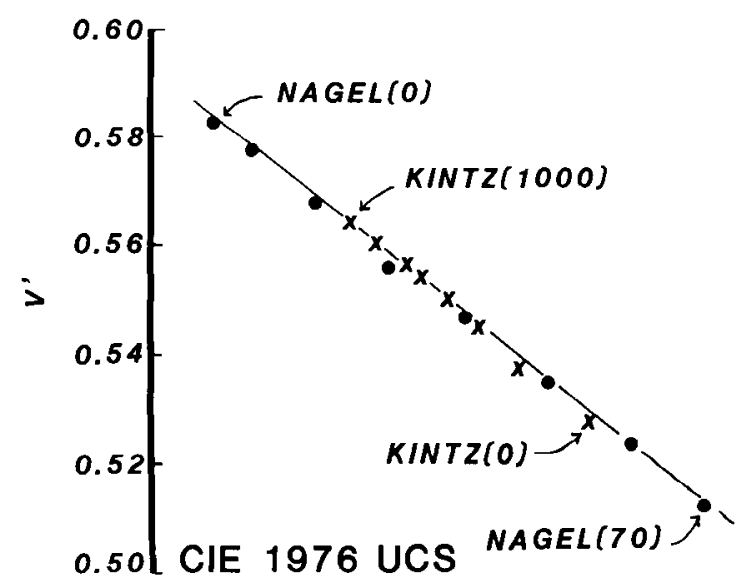

$$
\begin{array}{llllll}
0.1 & 0.2 & 0.3 & 0.4 & 0.5 & 0.6 \\
& & & & & \\
& & & & &
\end{array}
$$

Figure 1. The chromaticities of selected scale readings on the Nagel and the Kintz anomaloscopes plotted in CIE 1976 UCS space. The solid line represents the long-wavelength spectrum locus.

\section{Procedure}

The protanopes and deuteranopes (except K.L.) were pretested using either the American Optical (AO) or American Optical Hardy-Rand-Rittler (AO-HRR) pseudoisochromatic plates and the Farnsworth-Munsell 100Hue Test. Both tests were administered under CIE Illuminant $C\left(0.2 \mathrm{~cd} / \mathrm{m}^{2}\right)$, which also illuminated the neutral surface to which the observers adapted during anomaloscope testing. The observers were next tested on the two anomaloscopes. The bipartite field of the Kintz anomaloscope was viewed at a distance of about $25 \mathrm{~cm}$. For both anomaloscopes, observers were shown either the green or red primary field and were allowed to adjust the intensity of the yellow comparison field to obtain a complete color match or, failing that, a brightness match. Various red-green ratios were then presented by alternating between the red and green extremes thus establishing the matching range. For each red-green ratio, the observers were asked to fixate alternately the center of the bipartite field and an adapting surface also illuminated by Illuminant $\mathrm{C}$. The observers were instructed to determine whether the two fields matched exactly within $2 \mathrm{sec}$ after fixation. Approximately half of the observers were tested first on each instrument. A retest was then performed on the Kintz anomaloscope, in most cases on the next day, but always within 2 days of the original session. All testing was done monocularly using the preferred eye. Observers who wore untinted glasses or contact lenses were allowed to use them during testing.

Eighty-three normal observers were tested so that we could establish norms for the Kintz $(n=43)$ and Nagel $(n=40)$ anomaloscopes. The normal observers were classified using one of the two AO pseudoisochromatic plate tests. MMPs and MRs were obtained on both anomaloscopes using a method of limits procedure. The observers were presented with successive red-green primary settings and were asked to obtain a match by adjusting the luminance of the yellow comparison stimulus. The MR was first estimated by alternately presenting various primary stimuli on either side of the suspected MMP. Each end point of the MR was then more precisely estimated by presenting test stimuli in the vicinity of the end point. Each stimulus was presented from 4 to 6 times, and the end point was the one that matched the yellow comparison stimulus $50 \%$ of the time. The normal observers were also instructed to alternate their gaze between the anomaloscope fields and a $0.2 \mathrm{~cd} / \mathrm{m}^{2}$ white surface with a color temperature approximating that of CIE Illuminant $\mathrm{C}$. They, too, were instructed to declare a match only when the anomaloscope fields appeared identical in color within $2 \mathrm{sec}$ of fixating their center.

\section{RESULTS AND DISCUSSION}

Shown in Table 1 are the protan/deutan classifications of the 36 color-defective observers based on the pseudoisochromatic plate test, the age of each observer, and each observer's error score on the 100 -hue test. 
Table 1

Screening Data for the Deutan (D) and Protan (P) Observers

\begin{tabular}{lccl}
\hline Subject & Age & $\begin{array}{c}\text { Farnsworth-Munsell } 100 \\
\text { Error Score }\end{array}$ & $\begin{array}{l}\text { AO-HRR } \\
\text { Classification }\end{array}$ \\
\hline 1. M.A. & 44 & 56 & Mild P/D \\
2. A.A. & 32 & 168 & Strong-D \\
3. J.L. & 37 & 100 & Medium-P \\
4. K.T. & 22 & 116 & Medium-P \\
5. R.F. & 52 & 156 & Strong-P \\
6. F.R. & 20 & 136 & Medium-P \\
7. J.D. & 39 & 210 & Strong-D \\
8. G.A. & 37 & 146 & Medium-D \\
9. P.N. & 30 & 66 & Medium-D \\
10. E.O. & 33 & 118 & Medium-P \\
11. L.D. & & 192 & Medium-D \\
12. S.S. & 33 & 208 & Medium-P \\
13. B.H. & 51 & 80 & Strong-P \\
14. M.T. & 31 & 208 & Strong-D \\
15. K.L. & 21 & & \\
16. M.S. & 28 & 132 & Medium-P \\
17. L.M. & 22 & 182 & Medium-P \\
18. P.B. & 28 & 104 & Mild-P \\
19. H.A. & 70 & 480 & Strong-D \\
20. R.P. & & 292 & Medium-D \\
21. J.H. & 32 & 136 & Medium-P \\
22. A.H. & 36 & 230 & Medium-D \\
23. G.O. & 24 & 170 & Medium-D \\
24. R.C. & 39 & 64 & Medium-P \\
25. G.D. & 42 & 120 & Medium-P \\
26. D.S. & 22 & 158 & Medium-D \\
27. G.H. & 26 & 120 & Strong-D \\
28. D.W. & 48 & 152 & Medium-P \\
29. D.P. & 29 & 232 & Medium-D \\
30. S.L. & 56 & 162 & Strong-D \\
31. D.N. & 30 & 100 & Medium-D \\
32. W.D. & 41 & 54 & Mild-D \\
33. J.R. & 31 & 172 & Mild-D \\
34. A.M. & 42 & & Mild-D \\
35. H.F. & 39 & Medium-P \\
36. P.S. & 55 & Strong-D \\
\hline
\end{tabular}

\section{Comparison of Classifications}

To compare the Kintz and Nagel anomaloscopes, we adopted, with minor modifications, the criteria for classifying red-green color-defective observers proposed by Lakowski (1969) and by the NRC Committee on Vision (1981). Our own normative data were used to establish five categories (not all-inclusive) of red-green color defect, based on a standard Rayleigh color match, as follows: (1) color deviant (CD)-a normal MR, but an MMP between 1.5 and 3 standard deviations on either side of the normal mean; (2) color weak (CW)-an MR between 1.5 and 3 standard deviations on either side of the normal mean, and an MMP within \pm 1.5 standard deviations of the normal mean; (3) simple anomalous trichromat (SA) - an MR within \pm 2 standard deviations of the normal mean; (4) extreme anomalous trichromat (EA)-an MR exceeding \pm 2 standard deviations from the normal mean, and an MMP exceeding \pm 3 standard deviations from the normal mean; (5) dichromat (D)-an MR encompassing both primaries.

Each of the 23 observers classified as dichromatic on the Nagel anomaloscope were also classified as dichromatic on both the test and retest trials on the Kintz anomaloscope. The classifications of the 5 protanomalous and 8 deuteranomalous observers of the present study, based on data obtained using the Kintz and Nagel anomaloscopes, are shown in Table $2 .^{2}$ The two entries for the Kintz anomaloscope represent the test and retest conditions. The MR is expressed as a distance in UCS space (i.e., $\triangle E$ ), and the MMP is given by its UCS coordinates.

As suggested by the NRC Committee on Vision (1981), the validity of the Kintz anomaloscope as a color vision test was assessed by comparing it to the standard Nagel anomaloscope. The $\hat{K}$-statistic (Bishop, Fienberg, \& Holland, 1975) was used as a statistical measure of the agreement between the two tests in classifying color-defective observers. The $\hat{K}$-statistic represents the number of agreements between the two tests divided by the number of possible agreements, with the resulting value adjusted by the number of agreements expected by chance, as follows:

$$
\begin{aligned}
& \% \text { of observations for } \quad \% \text { of agreement } \\
& \hat{K}=\frac{\text { which there is agreement }-}{- \text { expected by chance }}
\end{aligned}
$$

A summary of the computed $\hat{K}$-statistics for the comparison of the Kintz and Nagel anomaloscopes (validity test) and the comparison of the test and retest on the Kintz anomaloscope (reliability test) is presented in Table 3. As may be expected, the validity and reliability were highest (in fact, perfect) for the simplest discrimination, namely that between anomalous trichromats (AT) and dichromats (D). For this test, categories CD, CW, SA, and EA were subsumed under the classification AT. The second row in Table 3 shows the $\hat{K}$-statistics for classification into the five categories defined earlier (i.e., CD, CW, SA, EA, and D). The $\hat{K}$-statistics for both the validity test $(0.84)$ and reliability test $(0.94)$ were somewhat reduced but were still very high. Finally, to assess the validity and reliability of the Kintz anomaloscope for a more difficult discrimination among nondichromatic color defectives, we performed an analysis excluding the 23 dichromats. Under these conditions (third row in Table 3), the extent of agreement in the validity comparison was reduced to 0.41 , whereas it was reduced to 0.62 in the test-retest comparison. It must be stressed that these latter $\hat{K}$-values are lower due to the restrictive criteria by which they were obtained. That is, they represent classification by degree of defect over four levels (CD, CW, SA, and EA) in a clinical population tested only once on each instrument. We conclude, based on the data of Tables 2 and 3, that the Kintz anomaloscope is an acceptable alternative to conventional anomaloscopes for classifying color-defective observers.

\section{SUGGESTIONS FOR IMPROVING THE KINTZ ANOMALOSCOPE}

Since spectroradiometers are relatively expensive and often unavailable, anomaloscopes are usually calibrated 
Table 2

Classifications, Matching Ranges $(\Delta \mathrm{E})$, and Matching Midpoints $\left(u^{\prime}, v^{\prime}\right)$ of the 13 Anomalous Trichromats, Each Tested Once on the Nagel Anomaloscope and Twice on the Kintz Anomaloscope

\begin{tabular}{|c|c|c|c|c|c|c|c|c|c|}
\hline & \multicolumn{3}{|c|}{ Kintz \#1 } & \multicolumn{3}{|c|}{ Nagel } & \multicolumn{3}{|c|}{ Kintz $\# 2$} \\
\hline & Class & MR & MMP & Class & MR & MMP & Class & MR & MMP \\
\hline \multicolumn{10}{|c|}{ Protanomalous } \\
\hline J.L. & $\mathrm{CW}$ & .021 & $.289, .556$ & SA & .303 & $.353, .545$ & & & \\
\hline P.B. & EA & .214 & $.341, .5$ & EA & .262 & $.441, .532$ & EA & .194 & $.330, .549$ \\
\hline R.C. & EA & .084 & $.327, .550$ & SA & .030 & $.525, .519$ & EA & .057 & $.369, .543$ \\
\hline D.W. & $\mathrm{EA}$ & .125 & $.364, .544$ & SA & .0385 & $.582, .511$ & EA & .173 & $.340, .547$ \\
\hline H.F. & EA & .200 & $.333, .549$ & EA & .400 & $.305, .552$ & EA & .168 & $.318, .551$ \\
\hline
\end{tabular}

Deuteranomalous

\begin{tabular}{llllllllll} 
M.A. & EA & .019 & $.255, .561$ & EA & .118 & $.201, .568$ & EA & .035 & $.258, .560$ \\
K.L. & EA & .066 & $.267, .559$ & EA & .268 & $.240, .563$ & & & \\
L.D. & EA & .044 & $.257, .561$ & EA & .299 & $.255, .560$ & EA & .067 & $.268, .559$ \\
L.M. & EA & .053 & $.261, .560$ & EA & .147 & $.180, .572$ & EA & .075 & $.275, .558$ \\
G.H. & EA & .078 & $.273, .558$ & EA & .132 & $.173, .573$ & SA & .013 & $.258, .560$ \\
J.R. & SA & .010 & $.260, .560$ & SA & .014 & $.228, .564$ & SA & .006 & $.258, .560$ \\
A.M. & EA & .060 & $.264, .559$ & EA & .074 & $.209, .567$ & EA & .049 & $.259, .560$ \\
W.D. & EA & .055 & $.262, .560$ & EA & .095 & $.234, .566$ & EA & .058 & $.264, .560$ \\
\hline
\end{tabular}

Note-Class $=$ classification; MR $=$ matching range; MMP $=$ matching midpoint.

by measuring the luminances of the primaries and the comparison field. Such a calibration would be difficult to perform on the Kintz anomaloscope, because the timer circuitry as presently designed (see IC1 in Figure 2 of Kintz, 1983) does not allow either the red or green primary to be presented alone. The relative proportion of the red and green primaries, and hence the color of the comparison stimulus, is determined by the duty cycle of the oscillatory square-wave output of ICl. We have determined by visual inspection that a duty cycle of between $0.1 \%$ and $0.3 \%$ is required for each primary to appear as if it alone were on. Kintz (1983) claimed that his circuit allows a duty cycle of about $0.01 \%$; if true, this would be sufficient to effectively present either primary alone. We have duplicated this circuit and found, however, that the resistance ratio used by Kintz (i.e., $R_{2} / R_{1}=10$ ) allows for a duty cycle of $1 \%$ at best and results in a perceptible narrowing of the chromaticity range of the LED primaries. Increasing the value of $R_{2}$ extends the duty cycle but also results in substantial variations in the output frequency. We suggest, therefore, that Kintz's IC1 be replaced by a conventional dual-timer circuit. ${ }^{3}$ Such a circuit requires fewer discrete components, provides for independent control of output frequency and duty cycle, and allows duty cycles of $0.1 \%$ or less.

Table 3

A Summary of the Computed $\hat{K}$-Statistics for Progressively More Difficult Discriminations Among the Color-Defective Observers of the Present Study

\begin{tabular}{|c|c|c|}
\hline & Validity & Reliability \\
\hline & $\overline{\text { (Kintz vs. Nagel) }}$ & $\overline{\text { (Kintz vs. Kintz) }}$ \\
\hline 2 Classes (AT vs. D) & 1.00 & 1.00 \\
\hline 5 Categories (Ds Included) & .84 & .94 \\
\hline 4 Categories (Ds Excluded) & .41 & .62 \\
\hline
\end{tabular}

Note-AT $=$ anomalous trichromats (includes categories $\mathrm{CD}, \mathrm{CW}, \mathrm{SA}$, and EA). D = dichromats (category D).
The precision with which MRs and MMPs can be measured depends largely on the spectral bandwidth and the spectral separation of the anomaloscope primaries (Pokorny \& Smith, 1984). The spectral bandwidths of the LED-derived primaries of the Kintz anomaloscope are somewhat greater than those of the Nagel anomaloscope primaries. The data of Figure 1, however, indicate no significant reduction in primary saturation relative to the Nagel anomaloscope, so spectral bandwidth is probably not a significant limiting factor in obtaining MRs and MMPs with the Kintz anomaloscope. A potentially more serious limitation is the spectral separation of the bicolor light bar LEDs. The peak wavelength of the red-LED primary is $635 \mathrm{~nm}$, whereas that of the green is $565 \mathrm{~nm}$, and the result is less separation of these primaries in chromaticity space as compared with those of the Nagel anomaloscope (see Figure 1). Since extreme anomalous trichromats can be distinguished from dichromats only by the extent of their matching range, a reduced separation of the anomaloscope primaries may be expected to result in the misclassification, relative to the standard Nagel anomaloscope, of some anomalous trichromats as dichromats. Such a misclassification never occurred in the present study, although this may be a fortuitous consequence of the limited matching ranges exhibited by our extreme anomalous trichromats. The problem of primary separation may be ameliorated somewhat in future implementations of the Kintz design by the use of a new bicolor light bar (Hewlett-Packard HLMP-2980) whose green component peaks at about $556 \mathrm{~nm}$ (R. Kintz, personal communication, November, 1986). For the present, however, we must conclude that the potential exists for the misclassification of extreme anomalous trichromats as dichromats by the Kintz anomaloscope. Pokorny and Smith (1984) have concluded that LED-based anomaloscopes require intensive validation. We agree and would 
further caution against a casual generalization of the present results and conclusions to other solid-state anomaloscopes. ${ }^{4}$

\section{REFERENCES}

Bishop, Y. M., Fienderg, S. E., \& Holland, P. W. (1975). Discrete multivariate analysis: Theory and practice. Cambridge, MA: MIT Press.

Dain, S. J., Strange, G., \& Boyd, R. (1980). A solid state anomaloscope. In G. Verriest (Ed.), Colour vision deficiencies V (pp. 181183). Bristol, England: Hilger.

GERI, G. A., \& NERI, D. F. (1987). Calibration and validation of a solid-state anomaloscope. Cooperative Study Series: AFHRL-TR-875, Air Force Human Resources Laboratory, Brooks Air Force Base, TX, and NSMRL Report No. 1086, Naval Submarine Base New London, Groton, CT.

KINTZ, R. T. (1983). A portable, solid-state anomaloscope. Behavior Research Methods \& Instrumentation, 15, 587-590.

LAKowSKI, R. (1969). Theory and practice of colour vision testing: A review. Part 2. British Journal of Industrial Medicine, 26, 265-288.

LAKOWSKI, R. (1971). Calibration, validation and population norms for the Pickford-Nicolson anomaloscope. British Journal of Physiological Optics, 26, 166-182.

NRC COMmittee on Vision. (1981). Procedures for testing color vision Report of Working Group 41). Washington, DC: National Academy Press.

Piantanida, T. P. (1976). A portable filter anomaloscope. Optical Engineering, 15, 325-327.

PoKorNy, J., \& SMITh, V. C. (1984). Metameric matches relevant for assessment of color vision. In G. Verriest (Ed.), Colour vision deficiencies VII (pp. 83-94). The Hague: Dr. W. Junk.

RAYLEIGH, LORD (1881). Experiments on colour. Nature. (London), 25, 64-66.

SAUnders, J. E. (1976). A red-green anomaloscope using light emitting diodes. Vision Research, 16, 871-874.
White, C. W., Wolbarsht, M. L., \& Tieger, T. (1975). A fast visual colorimeter. Behavioral Research Methods \& Instrumentation, 7 , 260-264.

\section{NOTES}

1. The solid-state anomaloscope used in the present study was the freeviewing model described by Kintz (1983, p. 590).

2. It should be noted that since the test stimuli and primaries of the two anomaloscopes are not spectrally identical, the MRs and MMPs shown for the two instruments may not be directly comparable. A direct comparison is not required for the purposes of the present study but could be made, according to Lakowski (1971), if the data of Table 2 were expressed in terms of equally perceptible units in UCS space.

3. Suitable circuits may be found, for instance, in Geri and Neri (1987), which is available upon request from the authors, or in the HewlettPackard Optoelectronics Designer's Catalog.

4. There are also several minor modifications of the Kintz (1983) anomaloscope that we would suggest in the interest of observer and experimenter convenience. Kintz recommends 10-turn potentiometers for controlling the primary ratio (i.e., red/green) and the luminance of the comparison stimulus. Several observers complained that too many turns were required to obtain a color match, and so we suggest using 3-turn potentiometers. Also, the turns-counting dials suggested by Kintz are unsuitable for data collection in semidarkness. We have constructed a solid-state anomaloscope using panel meters whose display intensity can be adjusted in accordance with the ambient illumination. Finally, in the absence of detailed information on the long-term stability of LED bicolor light bars, it would be prudent to periodically check the luminance of the three sets of LEDs used in the solid-state design. Test points for monitoring the various timer inputs to the light bars would greatly facilitate comparison of these inputs with the LED outputs as measured by an external detector.

(Manuscript received May 29, 1987; revision accepted for publication September 22, 1987.) 